\title{
Research on Education Quality Assessment Based on Campus Online Community
}

\author{
Yong-chao SHEN ${ }^{1, *}$ and Zhong $\mathrm{YAO}^{2}$ \\ ${ }^{1}$ Graduate School, Beihang University, Beijing, China \\ ${ }^{2}$ School of Economics and Management, Beihang University, Beijing, China \\ ${ }^{*}$ Corresponding author
}

Keywords: Campus online community, Graduate education, Quality assessment, PeopleRank.

\begin{abstract}
In recent years, the campus online community has become a new technology platform for gathering graduate students' attention. The discussion on how to use the campus online community for digging a new method to measure the graduate education quality has become a heated topic for scholars. This article measures the value-added process of individual human capital dynamically based on the PeopleRank model by tracking the graduate students' behavior on the campus based online community, such as knowledge sharing, online interactive learning exchanges, publishing academic papers, and ideological education dynamic reporting. The eigenvalue calculated by SNR matrix evaluates the quality of graduate education, and the results show that the eigenvalue can stably represent the quality of graduate education. Our study expands new theories and methods in the field of education quality assessment and provides new tools for quantifying graduate education quality assessment.
\end{abstract}

\section{Introduction}

Nowadays, with the rapid development of social online community, such as Facebook,Twitter, Linkedin, Groupon, Youtube, Weixin, Zhihu et al, colleges and universities have also built their own campus network community, Shuimu of Tsinghua university, Tianwaitian of Tianjin university, ihome of Beihang University are the successful representative of them. These network communities are used for students' management, ideological education, knowledge sharing, and academic innovation. Over the past two decades, along with the domestic graduate enrollment continues to expand. However, the scale of educational staff and supervisor team is relatively poor increased, these have led to the academic misconduct, ideological rigidity and poor guidance relationship.

The online community is also called a virtual community or an electronic community, which refers to people with common interests, hobbies, and needs to follow certain rules in a certain bounded network space and communicate through social software such as blogs, BBS, newsgroups, chat rooms, and emails ${ }^{[1]}$. It is a social community where participants focus on mutual communication, communication and interaction, and thus generate mutual closeness and recognition, and eventually form kinds of interpersonal circles ${ }^{[2]}$.

This article defines the campus online community as: officially recognized and formally registered, which have integrated the function of daily administration, research, teaching management, student ideological education, academic exchange and innovation, knowledge sharing et al, the platform which was used for daily affairs and daily interactions that can meet the needs of university teachers, graduate students, and educational staff ${ }^{[3]}$. It is a network system that realizes campus intelligence in smart internet environment, which enables users or virtual roles to acquire, publish, share, and manage various resources, assisting in knowledge innovation, collaborative work, problem solving, and decision support. As time accumulates, eventually everyone gains the value added ${ }^{[4]}$.

Why more and more scholars and users starting to pay attention to social online communities? To answer this question, we first need to look at what can be brought about through socialized communication and what new changes social networking online communities can bring to people's 
lives ${ }^{[5]}$. In the community, more information is obtained through the sharing of friends or classmates. The more social interactions are, the greater the probability of obtaining information outside its own areas of concern, and the acquisition of more information can positively promote the appreciation of the value of individual human capital ${ }^{[6]}$. When friends around you are all concerned about something or interacting in a certain network activity, the users will often be subtly influenced by this "Fashion" in the ordinary social communication in general. Reflected on the Internet, through a single click, you can see the recent concerns and research of friends or participate in social activities, so that the possibility of users participating in this activity or researching this issue is greatly increased, due to the circle of friends The cost of obtaining information is lower (the information reliability is stronger), and the possibility of solving the problem and then increasing the value of personal value is greater ${ }^{[7]}$.

By constructing the campus online community as defined in this article, students can dynamically track the value-added process of individual human capital, including knowledge sharing, online learning exchanges, participating interactive activities, published academic papers, and ideological education reporting. Based on the appreciation of capital value, we can evaluate the quality of graduate education.

\section{Method}

\section{Personal Utility Maximization Hypothesis}

Maslow's hierarchy of needs theory divides people's needs into physiological needs (PN), security needs (SN), love and belonging needs (LBN), respect and esteem needs (REN), self-actualization needs (SAN). For different people, because of their differences in material basis, growth experience, physical properties, social status, marital status, or demographic characteristics, the goal of life will be different for self-worth, and ultimately lead to differences in the level of combinational utility demand. We set the $\mathrm{u}(\mathrm{x})$ as a function of the demand level variables PN, SN, LBN, REN, and SAN, It can be seen that $\mathrm{u}(\mathrm{x})$ is a combination of requirements for the five demand variables Considering everyone's time distribution as a kind of consumption, the purpose of consumption is to satisfy all kinds of needs, it can be defined as:

$$
\text { Max: } u(x)=u\left(x_{P N}, x_{S N}, x_{L B N}, x_{R E N}, x_{S A N}\right), \text { s.t. } T=\sum_{i=1}^{5} t_{i} x_{i}
$$

Among them, $\mathrm{i}=1, \ldots, 5$ indicate the requirements of PN, SN, LBN, REN and SAN respectively, T is the overall time constraint, ti is the time allocation for satisfying various demand utility, so that the utility is maximized and the time constraint is satisfied. The available Lagrangian equation is:

$$
L=u\left(x_{i}\right)-\lambda\left(\sum_{i=1}^{5} t_{i} x_{i}-T\right)
$$

It is easy to see that for different requirements, the ratio of marginal utility is equal to the ratio of marginal time cost.

In fact, due to changes in the utility function of all people's needs, it will lead to different time allocation schemes. When a person's certain demand meets a certain level, he will not spend more time in this kind of demand. Therefore, under the constraints of the total time of a person's being known, according to its own ratio of time, we can figure out the combination of this person's needs in the demand dimension theory.In the campus online community, graduate students are affected by factors such as family environment, growth experience, and educational background. The distribution of time is bound to be different. For example, for the "rich second generation" and "official second generation" who have superior family conditions. The purpose of studying may be for the sake of REN and SAN, so that most time have been allocated to social communication and personal ability. For the "grassroots" of the poor family, the purpose of studying is to strive for more knowledge through their own efforts, and then change their own destiny. But in any case, they have social and 
emotional needs, which in a sense is precisely why they are willing to spend more time on the campus online community. For the personal perspective, they are all trying to maximize their personal utility. Of course, at different stages, the time allocation will be different ${ }^{[8]}$.

\section{The Essence of Postgraduate Education--Value Added of Human Capital}

When time is limited, everyone is always willing to choose the ability to use the skills with the highest value per unit time, the more people who are better at doing something, the more time will be allocated to the item. Ultimately, they are better at special skills, and the shortage of skills is even more rusty. From an economic and social point of view, employers want to find the best service providers with certain capabilities within reach. These demands eventually lead to an optimal skill in the individual's initial skill, leading to the inevitable division of labor in society. The biggest difference between education and the human capital market is that the purpose of education is to balance the development of individual capabilities on the basis of different personal skill.

Assume that all personal endowments in the campus online community can be summarized as $\mathrm{m}$ items, then each $n$ individual's skills are queuing according to their ability (the unit time value), and accordingly the unit time value of $\mathrm{n}$ individuals is taken as the standard value, then we can get $\mathrm{n}$ individuals' unit time value matrix on m skills.

It is not difficult to see that the essence of education is to fully explore and cultivate people's various aspects of capital value, thereby realizing the overall value-added of individual human capital.

\section{Value Added Mechanism of Individual Human in Campus online Community}

With the hypothesis of personal utility maximization and the analysis of the theoretical model of the essence of graduate education, based on the PeopleRank model, we further studied the value-added mechanism of individual human capital for graduate students in the campus online community. How to express the unit time value of a skill?

Assume that the directed graph $\mathrm{G}(\mathrm{V}, \mathrm{E})$ represents the network relationship among the users in the campus network community, where $V=\left\{V_{1}, V_{2}, \ldots, V_{n}\right\}$ represents the initial PR value set for each user, edge The set $\mathrm{E}=\{\mathrm{Eij}\}$ represents the time distribution probability.

The construction transition probability matrix $\mathrm{E}$ is as follows:

$$
E=\left(\begin{array}{ccc}
E_{11} & \cdots & E_{1 n} \\
\vdots & \ddots & \vdots \\
E_{n 1} & \cdots & E_{n n}
\end{array}\right)
$$

$\mathrm{E}_{\mathrm{mn}}$ represents the probability that the m node is linked to the $\mathrm{n}$ node, that is, the probability that users $\mathrm{m}$ and $\mathrm{n}$ exchange time values and share them. The vector $\mathrm{V}$ left multiplication matrix $\mathrm{E}$ once indicates that one iteration is completed $\left(\mathrm{V}^{1}=\mathrm{VE}\right)$, that is, one interaction is completed. This discrete state is a Markov chain in which the random transfer process is discrete time, the result of the $(n+1)$ is only related to the result of the $n$ time. Since $E$ is an $n \times n$ matrix, and $E_{i j}>0$. The transfer process is a regular chain, the above process must converge, therefore the PR value tends to be stable ${ }^{[9]}$.

Based on the initial integration ability of postgraduates at the time of enrollment, it is assumed that only the scores of the entrance examinations, retest scores, the overall level of published academic papers, personal aptitude and other personal aptitudes, and various social resources owned by the graduates are used to initialize individuals. The rankings and scores under various skills, we know that the more important people, the higher the unit time value, if he will share or interact with other people, the value increases accordingly. This interactive process can include academic exchanges in the online community, answers to difficult questions, amateur interest exchanges, and comments on published papers. With the rolling of the time axis, the interaction between users is frequent, the matrix transformation in the above model is frequently performed. Finally, due to the convergence of the process, the relative ranking of each graduate student can be obtained ${ }^{[10]}$. 


\section{Graduate Education Quality Assessment}

It is assumed that after the admission of graduate students, we initialize the PR matrix according to the influence factors of each graduate's different educational backgrounds, entrance exam scores, and retest scores. Here, we define people's various capabilities as people's ability tags, and construct a capability tag matrix $\mathrm{S}$ as follows for the $\mathrm{m}$ items of skills.

$$
S=\left(\begin{array}{ccc}
S_{11} & \cdots & S_{1 m} \\
\vdots & \ddots & \vdots \\
S_{m 1} & \cdots & S_{m m}
\end{array}\right)
$$

$\mathrm{S}_{\mathrm{ij}}$ is the correlation between skill $\mathrm{i}$ and $\mathrm{j}$, it is not a fixed value but a matrix that can be decomposed into several sub-capacity tags.

The relationship between people in the campus network community is constructed according to the directed graph $\mathrm{G}(\mathrm{E}, \mathrm{V})$. When the number of vertices is $\mathrm{n}$, the time-sharing relationship between people in the social network can be expressed as the following symbol label matrix.

$$
S S=\left(\begin{array}{ccc}
S S_{11} & \cdots & S S_{1 n} \\
\vdots & \ddots & \vdots \\
S S_{n 1} & \cdots & S S_{n n}
\end{array}\right)
$$

$\mathrm{SS}_{\mathrm{ij}}$ is the skill label of $\mathrm{i}$ share time to $\mathrm{j}$, similar to $\mathrm{S}, \mathrm{i}$ and $\mathrm{j}$ share skills may be more than one, $\mathrm{SS}_{\mathrm{ij}}$ is also a matrix, and $\mathrm{i}$ shares time with $\mathrm{j}$ on the label symbol of the matrix $\mathrm{SS}_{\mathrm{ij}}$, for example, $\mathrm{i}$ learns too many skills from $\mathrm{j}$.

Observing the matrix PR, S, SS, PR represents the PR value of the $n$ individuals in the social network, matrix $\mathrm{S}$ represents the layer structure of the $\mathrm{m}$ skills. The label matrix $\mathrm{SS}$ is the time transaction between people. It can be easily seen that the matrix PR is an $n \times m$ matrix, the matrix $S$ is an $m \times m$ matrix, and the matrix SS is an $n \times n$ matrix. Constructing a new $(n+m) \times(n+m)$ matrix through matrix transformation:

$$
A=\left|\begin{array}{ll}
S S & P \\
P^{T} & S
\end{array}\right|
$$

A is called the Social Networks Relationship Matrix. Based on the matrix transformation and matrix drilling and drilling, we can use the SNR matrix to represent various interactions that occur in the campus online community, such as the sharing of knowledge and skills, and the attention of academics in the subject area. It can be obtained by solving the eigenvalue of A, which is called a stable campus network community.

Assume that we can record the distribution of the time for each graduate student on the Internet 24 hours a day, and the label each unit time allocation, we can get the value of all elements in the person-to-person relationship label matrix SS. For example, if the "concerned" action is regarded as the minimum time unit, then A's attention to $\mathrm{B}$ must be based on a certain reason. Certain characteristic of B attracts A's attention, through the value of the relational label matrix SS, the element value of the capability matrix $\mathrm{P}$ can be calculated according to the PeopleRank model and the PR value of each member's ability. In this way, the specific SNR matrix can be determined. When assessing the quality of postgraduate education in different universities, the postgraduate education quality assessment is based on the eigenvalues of the SNR matrices found in each university. If there are multiple eigenvalues in a university's SNR matrix, there is a "spiral ascent" in the campus online community, which means that the quality of graduate education continues to increase; if there is only one characteristic value in a university's SNR matrix, the only steady state exists is that the quality of postgraduate education is in a stable development period; if there is no characteristic value, then the 
campus online community is considered "chaotic", that is, the quality of graduate education cannot be guaranteed.

\section{Conclusion}

Under the premise of the connotation of the campus online community defined in this article, based on the assumption of personal utility maximization, the essence of graduate education is analyzed, the process of personal human capital value appreciation. Based on this, we use the PeopleRank model algorithm, the value-added mechanism of individual human capital of graduate students in the campus network community is deduced. Finally, the quality of graduate education is evaluated through matrix construction and transformation. The research in this paper provides a tool to quantify the quality of postgraduate education assessment, and to a certain extent, it illustrates the reality and possibility of evaluating graduate education quality based on the campus network community. However, in actual operation, due to incomplete symmetry of information and different standards for initializing PR values, the PR value labels are misplaced, thereby affecting the final evaluation results.

\section{Acknowledgement}

This research was financially supported by the special research funds for graduate education and development of Beihang University (2017015).

\section{References}

[1] J.M. Sims, Communities of practice: Telemedicine and online medical communities, J. Technological Forecasting and Social Change. 126(2018) 53-63.

[2] J.H. Jin, Y.J. Li, X.J. Zhong, et al, Why users contribute knowledge to online communities: An empirical study of an online social Q\&A community, J. Information \& Mangement.52(2015) 840-849.

[3] C.M.Chiu, H.Y. Huang, H.L. Cheng, eta al, Understanding online community citizenship behaviours through social support and social identity, J. International Journal of Information Management. 35(2015) 504-519.

[4] K.Christensen, K H. Liland, K Kvaal, et al, Mining online community data: The nature of ideas in online communities, J. Food Quality and Preference. 62(2017) 246-256.

[5] C. Martin, Entrepreneurs Acting as Mentorsin Online Communities for Students Enrolled in MA Studies, J. Social and Behavioral Sciences.197(2015) 645-651.

[6] C.Teng, Impact of avatar identification on online gamer loyalty: Perspectives of social identity and social capital theories, J. International Journal of Information Management. 37(2017) 601-610.

[7] P. A. Pavlou, F Mendel, Understanding and Predicting Electronic Commerce Adoption: An Extension of the Theory of Planned Behavior, J. MIS Quarterly. 30(2006) 115-143.

[8] J.H.Chang, Y.Q.Zhu, S.W.Wang, et al, Would you change your mind? An empirical study of social impact theory on Facebook, J. Telematics and Informatics. 35(2018) 282-292.

[9] Sergey Brin, Lawrence Page. The Anatomy of a Large-Scale Hypertextual Web Search Engine, J. Computer Network and ISDN Systems. 30(2008) 107-117.

[10] F Pedroche, G E Garcia Romance, Sharp estimates for the personalized Multiplex PageRank, J. Journal of Computational and Applied Mathematics.330(2018) 1030-1040. 